\title{
Miasis oral en un paciente adolescente con déficit neurológico crónico
}

\author{
Adriana Marcela Arenas-Rojas* \\ Nathalia Andrea Barajas-Ramírez**

\begin{abstract}
* Estudiante de internado, XII nivel de Medicina. Escuela de Medicina. Facultad de Salud. Universidad Industrial de Santander. Hospital Universitario de Santander. Bucaramanga. Santander. Colombia.

** Médica y Cirujana. E.S.E. Hospital Regional de San Gil. San Gil. Santander. Colombia.
\end{abstract}

Correspondencia: Dra. Nathalia Andrea Barajas Ramírez. Dirección: Carrera 17 \#45-221. Portal II. Casa 23. Girón. Santander. Colombia. Correo electrónico: nathalia2305@hotmail.com

\section{RESUMEN}

La miasis en cavidad oral es una presentación poco frecuente de invasión por larvas de moscas, favorecida por ambientes cálidos y húmedos, en pacientes con malas condiciones sanitarias. El diagnóstico se realiza por visualización directa de las larvas que se agrupan en forúnculos y su tratamiento incluye medidas sistémicas y locales para la erradicación. Se presenta un caso en un paciente masculino de 14 años de edad con parálisis cerebral afectado por esta patología poco frecuente. MÉD UIS. 2015;28(3):381-5.

Palabras clave: Miasis. Boca. Mucosa Oral. Parálisis Cerebral. Higiene Bucal. Dípteros.

\section{Oral myiasis in an adolescent patient with chronic neurological deficit}

\section{ABSTRACT}

Myiasis in oral cavity is an infrequent presentation of invasion by fly larvae, favored by hot and humid environments, in patients with poor sanitation. Diagnosis is made by direct visualization of the larvae that cluster in boils. Treatment includes systematic and local measures for the eradication. A case of this infrequent pathology affecting a 14 year-old male patient with cerebral palsy is presented. MÉD UIS. 2015;28(3):381-5.

Keywords: Myiasis. Mouth. Cerebral Palsy. Oral Hygiene. Diptera.

¿Cómo citar este artículo?: Arenas-Rojas AM, Barajas-Ramírez NA. Miasis oral en un paciente adolescente con déficit neurológico crónico. MÉD UIS. 2015;28(3):381-5.

\section{INTRODUCCIÓN}

El término miasis se deriva de las palabras griegas "myia" que significa mosca e "iasis" que significa enfermedad; acuñado por Hope en 1840 y descrito por primera vez en 1909 por Laurance. En 1965, Zumpt definió la miasis como la infestación de tejidos vivos de animales o humanos por larvas de dípteros, las cuales se alimentan de los tejidos vivos o necróticos, sustancias corporales líquidas o comida ingerida, La miasis oral es una condición poco frecuente que hace referencia a la invasión del tejido de la cavidad oral por larvas de mosca. Hay un total de 54 reportes de miasis oral en las bases bibliográficas PubMed, Medline, CSIC, Scopus, Cochrane, LILACS, CINALH, BUS y BDDOC ${ }^{3-5}$. 
La miasis humana ocurre a nivel global, siendo un parasitismo endémico en regiones tropicales y semitropicales de América del Sur, el Caribe, Centroamérica, el Sudeste asiático, subcontinente Indio, África subsahariana y rara por encima de los 2000 metros de altura; el resto de casos se consideran brotes o parasitismos accidentales. Los países con mayor miasis oral son India y Brasil, con $28 \%$ y $26 \%$ de los casos respectivamente, posiblemente debido al ambiente cálido y húmedo que favorece el desarrollo de la enfermedad ${ }^{17}$.

Su vía de transmisión a huéspedes humanos varía entre las diferentes especies de moscas y larvas que causan la infestación, y la prevalencia de cada subespecie depende la distribución geográfica ${ }^{1-7}$. Las especies de moscas que causan miasis son Dermatobia hominis, Cochiliomyia hominivorax, Cordylobia anthropophaga, Sarcophaga, Calliphora, Lucilia, Gasterophilus. De estas, las más frecuentes y consideradas endémicas en Sudamérica son las dos primeras. A nivel mundial, las especies con mayor presencia son las larvas Cochliomyia hominivorax y Chrysomya bezziana, $28 \%$ y $13 \%$ respectivamente ${ }^{3,4-7}$. La Dermatobia hominis pertenece a la familia Oestridae o moscas del "nuevo mundo", su ciclo vital oscila entre 120 y 122 días, y afecta específicamente humanos, ganado bovino, ovino, gatos, perros y conejos. Se cree que $D$. hominis escapa de la destrucción del sistema inmune por medio de la supresión del sistema humoral durante la infestación $n^{1,3-6}$.

El ciclo vital de la mosca inicia con la etapa de huevo, seguida por el estadio de larva, el estadio de pupa y finalmente la mosca adulta. Las condiciones necesarias para depositar los huevos y la supervivencia de las larvas son la humedad, la presencia de tejido necrótico y una temperatura adecuada. La etapa de larva dura de seis a ocho días, durante la cual son patógenas para los humanos. Las larvas son fotofóbicas, por lo tanto, tienden a ocultarse de forma profunda en los tejidos, lo cual también les permite asegurar un nicho apropiado para pasar a la etapa de pupa ${ }^{2}$.

Se clasifica dependiendo de la condición del tejido involucrado como miasis accidental, cuando las larvas son ingeridas con la comida; miasis semi-específica, cuando las larvas son depositadas en el tejido necrótico de una herida; y miasis obligatoria, cuando las larvas afectan los tejidos sanos. Además, puede ser clasificada según la región anatómica comprometida como miasis cutánea, miasis de orificios externos y miasis de órganos internos. Según las características de las larvas puede ser clasificada como primaria o secundaria. La miasis primaria, también llamada "obligatoria", es causada por larvas biontófagas, que se alimentan de tejidos vivos; por otro lado, la miasis secundaria, también llamada "facultativa", es causada por larvas necrobiontófagas que se alimentan de tejidos muertos ${ }^{1,3-8}$.

Los sitios anatómicos más comúnmente afectados por miasis son ojos, heridas cutáneas, senos paranasales, oídos, pulmones, intestinos, vesícula biliar, vagina, cavidades nasales, y de forma menos frecuente, la cavidad oral ${ }^{1,2}$. De las miasis cavitarias, las miasis orales posiblemente son las más frecuentes, ubicándose mayoritariamente en la región gingival superior $(22 \%)^{4-6}$.

La miasis en cavidad oral presenta mayor prevalencia en pacientes con necesidad de cuidados especiales, como aquellos con alteraciones mentales y físicas, trastornos neurodegenerativos, parálisis cerebral, dificultades para el aprendizaje, postración en cama y adultos mayores; siendo el déficit neurológico crónico la comorbilidad más frecuente $(39 \%)^{2-6}$. Esto se relaciona con los múltiples factores predisponentes presentes en ellos, como lo son la pobre higiene bucal, incompetencia labial, halitosis, respiración bucal durante el sueño, diabetes, lesiones ulcerativas y carcinomas en piel, trastornos convulsivos y traumas faciales o en los tejidos de la cavidad oral con heridas expuestas al ambiente y a hipersalivación ${ }^{1,2,9}$. En cuanto al género, los hombres son los que tienen mayor número de casos $(72 \%)^{3-5}$.

Se presenta un caso clínico de miasis en cavidad oral en un adolescente inicial con parálisis cerebral, ocurrido en una región rural de Santander, Colombia, con el fin de resaltar la importancia del reconocimiento de esta patología, así como la adecuada higiene oral y protección bucal en pacientes con trastornos que afectan su estado de conciencia. La divulgación de la historia clínica y las fotografías se realizó previa autorización de la madre del menor.

\section{Presentación de caso}

Paciente masculino de 14 años de edad, con antecedente de meningitis al año de nacimiento, con parálisis cerebral y síndrome convulsivo secundario, en tratamiento con ácido valpróico, quien es llevado al hospital de su municipio de residencia por un cuadro clínico de aproximadamente un día de evolución de 


\section{SEPTIETRBRE-DICIEMBRE}

rechazo a la vía oral asociado a hemorragia gingival en incisivos superiores. Al ingreso se encuentra paciente con parálisis motora generalizada, hidratado, tolerando dieta líquida, al examen bucal se destaca estado de salud oral deficiente, hiperplasia gingival medicamentosa y piezas dentales temporales en mal estado. Adicionalmente, se observan 11 abscesos de larvas distribuidos de la siguiente manera: dos en región vestibular de incisivos superiores, tres en región vestibular de incisivos inferiores, tres en región palatina de incisivos superiores y tres en región lingual de incisivos inferiores (Ver Figuras 1 y 2).

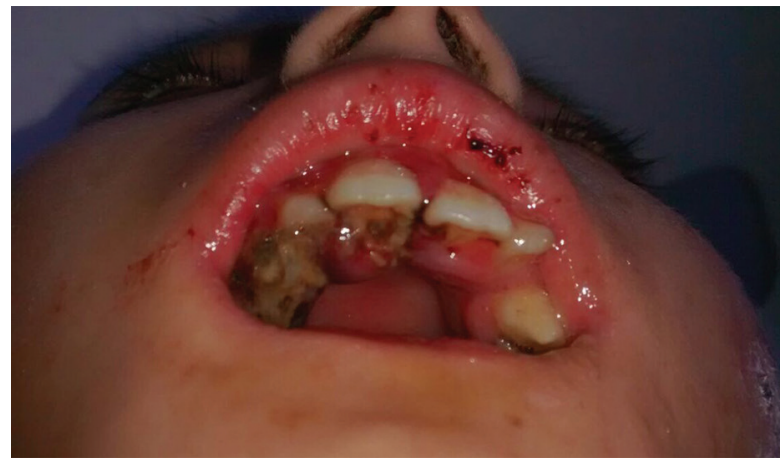

Figura 1. Abscesos de larvas en región anterior de la cavidad oral Fuente: Autor.

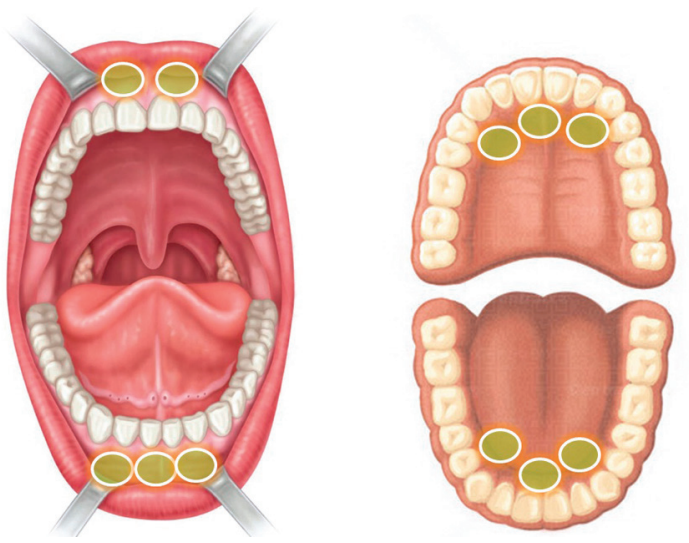

Figura 2. Distribución de los 11 abscesos de larvas encontrados en la cavidad oral: 2 en región vestibular de incisivos superiores, 3 en región vestibular de incisivos inferiores, 3 en región palatina de incisivos superiores y 3 en región lingual de incisivos inferiores Fuente: Autor

Se procede a realizar limpieza oral con solución salina normal, gasas, uso de vaselina como agente asfixiante en cada absceso y extracción de larvas con pinzas para un total de 30 parásitos, posteriormente se realiza retiro de dientes deciduos (Ver Figura 3) y se da salida con recomendaciones de uso de tapabocas, toldillo e higiene oral. Asiste a control a los tres días para continuación del tratamiento, se realizan iguales medidas de limpieza y se extraen dos larvas adultas, una pequeña y demás residuos de dientes temporales. Durante la tercera visita realizada a los ocho días, se comprueba la ausencia de larvas y se observa adecuada evolución de cicatrización e higiene oral. No se realizó identificación del agente etiológico, dada la limitación geográfica para el estudio y que este no es finalmente el objetivo de la presentación del caso.

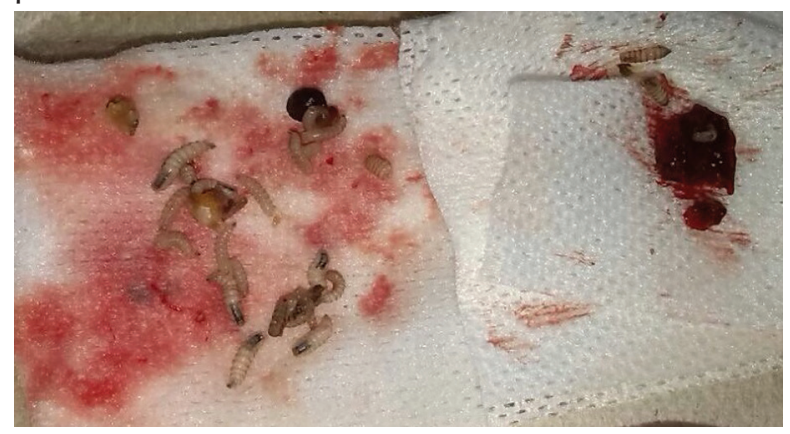

Figura 3. Larvas de díptero extraídas de cavidad oral Fuente: Autor.

\section{Discusión}

La miasis oral es una condición poco frecuente que hace referencia a la invasión del tejido de la cavidad oral por larvas de mosca y puede ser clasificada como miasis facultativa y miasis obligatoria, esta última haciendo referencia a larvas que se alimentan de tejido vivo como es el caso del caso presentado ${ }^{1,3-8}$. De acuerdo a lo reportado en la literatura, la miasis ocurre principalmente en personas de género masculino, adultos mayores o en pacientes con enfermedades neurológicas, existiendo una mayor prevalencia en las regiones de clima caliente, en personas que proceden de lugares con malas condiciones higiénicas y con un nivel socioeconómico bajo ${ }^{2,5-10}$. Similar al caso presentado, que corresponde a un adolescente con parálisis cerebral habitante de una zona rural en la cual la presencia de discapacidad mental, el estrato socioeconómico bajo y la respiración bucal fueron los principales factores predisponentes para el desarrollo de miasis oral.

Las manifestaciones orales de la infección incluyen edema, eritema y dolor pulsátil debido al movimiento de las larvas o a las aperturas hechas al margen de las superficies gingivales, donde se pueden observar las mismas ${ }^{6-10}$. En el caso presentado, la localización de las lesiones en la parte anterior de la cavidad oral sugiere inoculación directa de los tejidos. Similar a este, los casos reportados en la literatura se ubicaron en paladar anterior, encías, labios o comisura labial, 
afectando principalmente a pacientes con factores predisponentes adicionales como incompetencia labial, mordida abierta y respiración bucal ${ }^{6,9,10}$.

El diagnóstico clínico suele ser fácil de realizar por la aparición de larvas en los tejidos, con excepción de algunos casos donde estas se encuentran ubicadas debajo de la piel. Sin embargo, la determinación de la causa etiológica no es un trabajo sencillo y debe ser hecho por personal experto dado que esto precisa los condicionantes de la infestación; en el caso presentado en esta publicación no fue posible determinar la etiología dado a dificultades geográficas para su estudio. La mayoría de moscas causantes de miasis son atraídas a depositar sus huevos o larvas en respuesta a los olores de los tejidos necróticos, por esto los pacientes con heridas supurativas o crónicas tienen un riesgo particular de presentar miasis ${ }^{3-8}$.

El manejo de la miasis oral debe tener como objetivo la erradicación de las larvas, esto puede incluir medidas sistémicas y locales. Los tratamientos sistémicos incluyen antibióticos de amplio espectro como ampicilina o amoxicilina, que se utiliza como medida profiláctica o cuando la herida presenta infección secundaria ${ }^{3,9,11}$, sin embargo, no se encuentra en la literatura un esquema claro para el manejo de esta patología. En los casos más severos también se ha reportado el uso de tratamiento sistémico con ivermectina, un macrólido semisintético, administrado en dosis única de 150-200 $\mathrm{mg} / \mathrm{kg}^{9-11}$. Las medidas locales consisten en el retiro quirúrgico de las larvas acompañado de un adecuado desbridamiento de los tejidos necróticos, asociado o no a la administración de medicamentos asfixiantes o sustancias irritantes que obligan a las larvas a salir, seguida de irrigación con solución salina tibia' ${ }^{7,9,11}$. Es importante retirar todas las larvas, de lo contrario la cavidad afectada no sanará correctamente y puede quedar crónicamente infectada. De igual manera, se debe evitar la ruptura de las larvas, ya que esto puede generar una reacción de cuerpo extraño $0^{5,8-13}$. Teniendo en cuenta la importancia del manejo local y la ausencia de esquemas para el manejo antibiótico sistémico, en el caso presentado se realizaron solo medidas de retiro mecánico y limpieza, obteniéndose resultados satisfactorios.

\section{CONCLUSIÓNES Y RECOMENDACIONES}

En base al caso previamente expuesto y en aras de dejar al profesional medico unas recomendaciones básicas para el abordaje a partir de la experiencia del caso anterior se puede definir que la miasis en cavidad oral es una situación poco frecuente que se favorece por condiciones climáticas de calor y humedad. Se presenta mayoritariamente en pacientes con pobre higiene bucal, halitosis y respiración bucal al igual que en pacientes con patologías neurológicas de base y adultos mayores, predominando en el género masculino.

El diagnóstico clínico es fácil de determinar mediante la presentación clínica tras la visualización de las larvas en el tejido afectado, las cuales son atraídas por el olor del tejido necrótico, y se presentan como lesiones de tipo forúnculo y gusaneras. Sin embargo, el diagnóstico etiológico requiere pericia del especialista para su correcta determinación. Las manifestaciones en cavidad oral se caracterizan por presencia de eritema, edema, dolor secundario al movimiento de las larvas y la ubicación suele ser en la cavidad anterior de la boca, sugiriendo inoculación directa.

El tratamiento incluye medidas sistémicas como manejo antibiótico de amplio espectro y medidas locales que consisten en la extracción de las larvas y el desbridamiento del tejido necrótico, asociado o no a sustancias asfixiantes o irritantes que obligan a las larvas a salir de la cavidad. En casos severos se indica el uso de ivermectina, un macrólido que causa parálisis y muerte de las larvas.

Finalmente, y a manera de conclusión la miasis oral no es una condición frecuente y muchos profesionales de la salud desconocen su presentación clínica y su progresión, lo cual puede llevar a retrasos en el tratamiento y la aparición de complicaciones en algunos casos; es por esto que la prevención es la medida más importante. La miasis en la región orofacial se puede prevenir educando a las personas procedentes de áreas rurales y grupos socioeconómicos bajos acerca de la higiene personal, cuidado de las heridas, control de la población de dípteros y mantenimiento de medidas sanitarias, mediante la limpieza de los posibles sitios donde pueden proliferar y educando a los individuos que habitan lugares sin sanidad básica, quienes presentan un mayor riesgo. Se debe prestar atención especial a los pacientes en condición de discapacidad mental, física o de aprendizaje. Muchos cuidadores no tienen el conocimiento necesario para reconocer la importancia de la higiene oral y no practican una apropiada limpieza a este tipo de pacientes. En 
casos severos, los padres o cuidadores deber recibir asesoría acerca de la importancia de que las personas a su cargo mantengan una adecuada salud oral.

La prevención constituye una de las estrategias más significativas frente a esta patología, se resalta la importancia de brindar educación acerca de higiene personal, cuidado de heridas, control de la población de dípteros y mantenimiento de medidas sanitarias a personas procedentes de áreas rurales y grupos socioeconómicos bajos quienes tienen un mayor riesgo.

\section{Referencias BibLIOgRÁficas}

1. Sharma A. Oral myasis is potential risk in patients with special health care needs. J Global Infect Dis. 2012?4:601.

2. Reddy MHR, Das N, Vivekananda MR. Oral myiasis in children. Contemp Clin Dent. 2012;3(Suppl1):S19-22.

3. Reinoso-Quezada S, Alemán-Iñiguez JM. Rara miasis maxilar por Cochliomyia hominivorax. Reporte de caso, actualidad y entomología. Rev Esp Cir Oral Maxilofac. 2014:1-5.
4. Azoubel A, de Santana T, Linard R, Carneiro E, Macedo B, Rodrigues J. Oral and maxillofacial myiasis: a case series and literature review. Oral Surg Oral Med Oral Pathol Oral Radiol Endod. 2011;112:e81-5.

5. González C, Salamanca JC, Olano V, Pérez CE. Miasis cavitaria. Reporte de un caso. Rev. Fac. Med. 2008;16(1):95-8.

6. Avula JK, Avula H, Arora N, Manchukonda UK, Vivekavardhan Reddy N. Orofacial myiasis of the gingiva and nasal cavity: a report of two cases and general review. J Periodontol. 2011;82(9):1383-8.

7. Daltoé FP, Nosé AR, Nosé FR, Vanti LA, Mosca RC, Mantesso A. Oral myasis in two children. The Pediatric Infectious Disease Journal. 2013;32(5):572-3.

8. Zuñiga IR. Miasis: un problema de salud poco estudiado en México. Revista de Enfermedades Infecciosas en Pediatría. 2009;22(88):121-5.

9. Bhagawati BT, Gupta M, Singh S. Oral myiasis: A rare entity. Eur J Gen Dent. 2013;2:312-4.

10. De Conto F, Moura M, Furlanetto TM, Marcolin D, Stobbe JC, Rovani G, et al. The Use of Ivermectin in the Adjunct Treatment of Oral Cavity Myiasis. Acta stomatol Croat. 2013;47(3):246-52.

11. Raffaldi I, Scolfaro C, Pinon M, Longo S, Savoia D, Tovo PA. A strange gingival swelling in an Italian child: a case of oral myiasis. Le Infezioni in Medicina. 2013;1:56-9.

12. Saravanakumar MS, Vasanthakumari A, Bharathan R. Oral health status of special health care needs children attending a day care centre in Chennai. Int J Stud Res. 2013;3:12-5.

13. Eseigbe EE, Taju NF, Lateef ST. Challenges in care of the child with special health care needs in a resource limited environment. J Neurosci Rural Pract. 2013;4:204-6. 